\title{
FEATURES OF BACKSCATTER IONOSPHERIC SOUNDING AS STUDIED WITH A CHIRP IONOSONDE DOI: 10.12737/23509
}

\author{
S.N. Ponomarchuk \\ Institute of Solar-Terrestrial Physics SB RAS, \\ Irkutsk,Russia,spon@iszf.irk.ru \\ V.I. Kurkin \\ Institute of Solar-Terrestrial Physics SB RAS, \\ Irkutsk,Russia,kurkin@iszf.irk.ru
}

\author{
M.S. Penzin \\ Institute of Solar-Terrestrial Physics SB RAS, \\ Irkutsk,Russia,penzin@iszf.irk.ru
}

\begin{abstract}
We present the results of the studies of backscatter ionospheric sounding (BS) on the basis of a multipurpose chirp ionosonde developed in ISTP SB RAS. We analyze BS experimental data obtained during different seasons from 2005 to 2009. The accumulated dataset allows us to investigate features of BS signal propagation in various heliogeophysical conditions. To analyze and interpret BS signals on ionograms, we use the results of modeling of characteristics for chirp signals in the backscatter and oblique ionospheric sounding under a waveguide approach. We have revealed the most characteristic types of ionograms and have established conditions of appearance of a given type depending on the time of day, season, sounding direction, and medium
\end{abstract}

\section{INTRODUCTION}

The backscatter ionospheric sounding (BS) method has been adopted for ionospheric research along with vertical and oblique sounding methods. It has been used for predicting conditions of radio wave propagation in radio paths and over-horizon radar systems. As regards the transmitter power, we can distinguish radiotechnical systems of two extreme types, which operate in the backscatter sounding mode. The first type includes systems with a transmitter power exceeding $10 \mathrm{~kW}$; the second type comprises systems with a power less than $1 \mathrm{~kW}$. The quality of BS ionograms depends directly on the transmitter power: the higher is the transmitter power, the greater is the signal-to-noise ratio. This allows us to detect high-resolution scattered signals from long ranges and to improve the quality of ionogram processing and interpretation. However, a high transmitter power increases costs for maintenance of the system. Moreover, a problem arises of electromagnetic compatibility of the sounding system as a whole. To solve the problem of increasing the power potential of stations, while limiting the transmitter power, and to provide high time resolution, radars extensively use chirp signals (Varakin, 1970; Cook, Bernfeld, 1971; Sinnott, 1988; Wise, 2004; Earl, Ward, 1987]. In ionospheric studies, chirp signals are exploited to devise ionosondes operating in vertical, oblique, and backscatter sounding modes [Brynko et al., 1988; Ivanov et al., 2003; Podlesny et al., 2013]. Up-to-date chirp oblique sounding ionosondes detect signals over long paths, including round-the-world ones [Ivanov et al., 1997; Kurkin, 2000; Ivanov et al., 2003]. A new instrument - a chirp ionosonderadio- conditions. In winter, spring, and autumn, the prevailing types of ionograms are those with BS signals corresponding to the propagation mode through reflection from the $\mathrm{F}$ layer. Signals reflected by $\mathrm{E}$ or $\mathrm{E}_{\mathrm{s}}$ layers are recorded during summer periods. At the same time, frequencies of the received signals are sufficiently large, and sometimes there are no reflections from the $\mathrm{F}$ layer.

Keywords: ionosphere, ionogram, radio wave propagation, backscatter ionospheric sounding. direction finder - has been developed, which can simultaneously measure the key characteristics of the ionospheric channel (distance-frequency, amplitude-frequency, and angular-frequency characteristics) in the entire frequency range of HF radio signal propagation. This instrument was used in experimental studies of radio wave propagation along paths of various lengths and directions in the natural and artificially disturbed ionosphere. The ionosonderadiodirection finder can be applied to the over-horizon short-wave radar of Earth's ionosphere on the planetary scale [Valov et al., 2012; Vertogradov et al., 2013; Uryadov et al., 2013]. In the backscatter ionospheric sounding mode, the chirp ionosonde can detect signals scattered by Earth's surface at large distances from a transmitter under relatively low transmitter powers with the use of a signal frequency compression technology [Philipp et al., 1991; Ilyin et al., 1996; Ivanov et al., 2003]. The multi-year research on BS sounding with the chirp ionosonde, developed at ISTP SB RAS, has revealed that such systems can be applied to diagnosing the propagation medium within the maximum range of one hop $(\sim 3000-4000 \mathrm{~km})$ [Altyntseva et al., 1990; Ponomarchuk et al., 2009]. This paper reports results of a study of features of the backscatter ionospheric sounding in northeastern Russia. This study relies on experimental data obtained under various heliogeophysical conditions in 2005-2009. To interpret BS signals on ionograms, we have used results of the modeling of characteristics of chirp signals in the backscatter ionospheric sounding under the waveguide approach with the IRI model [Ponomarchuk et al., 2009; Bilitza, Reinisch, 2008; Ponomarchuk et al., 2014]. 


\section{EXPERIMENT AND DATA PROCESSING}

We analyze experimental BS data acquired with the ISTP SB RAS chirp ionosonde in different seasons in 2005-2009. A transmitter was in the town of Usolye Sibirskoye $\left(52.8^{\circ} \mathrm{N}, 103.3^{\circ} \mathrm{E}\right)$; a receiver, in the village of Tory, the Republic of Buryatia $\left(51.8^{\circ} \mathrm{N}, 103^{\circ} \mathrm{E}\right)$. The transmitter was a log-periodic antenna array with the azimuth rotary mechanism. The receiver was a BS-2 system of antennas with their pattern maximums directed at $55^{\circ}, 121^{\circ}, 235^{\circ}$, and $301^{\circ}$ azimuths. The transmitter power was less than $1 \mathrm{~kW}$, therefore we could detect scattered signals largely within the maximum range of one hop ( $3000-4000 \mathrm{~km})$. The BS studies were mainly carried out during the minimum of solar activity between solar cycles 23 and 24. During the experiments, geomagnetic conditions were quiet.

The secondary processing of ionograms had been made to remove noise components from the image and to extract points with significant amplitude corresponding to arrival times of a signal along the leading edge of the BS signal or to the maximum in the amplitude relief [Grozov et al., 2012; Grozov et al., 2013]. To eliminate noise from the image and to restore signal samples, we employ a median filter capable of smoothing noises, reducing the blurring of track boundaries, and restoring values in track breaks. To separate single artifacts, partially restore data, and identify the primary track on an ionogram, we use a cellular automaton mechanism. Signals extracted on ionograms during secondary processing are interpreted based on the results of modeling of BS signal characteristics. Experimental studies and modeling of propagation characteristics have established that the main contribution to BS signal formation is made by regions of scattering by Earth's surface located near the boundary of the illuminated zone (transition from the silent zone to the reception zone for oblique sounding signals) for individual hops, [Chernov, 1971; Altyntseva et al., 1990]. The position of the boundary of the illuminated zone is found using the distance-frequency characteristic (DFC) of BS signals along the leading edge because the maximum in the amplitude relief of a BS signal is close in the group path to the leading edge boundary [Dyson, 1991; Ponomarchuk et al., 2009]. Therefore, BS ionograms can be interpreted by calculating the frequency dependence of the minimum group path $P_{\mathrm{m}}(f)$ corresponding to the closing point of the upper and lower rays for oblique sounding signals. The operating frequency $f$ for the respective range $D_{\mathrm{m}}(f)$ is the maximum usable frequency (MUF) of radiocommunication. For BS DFC on ionograms to be automatically interpreted and restored, a method has been worked out which is based on the adiabatic dependence of the minimum group path of a signal scattered by Earth's surface in the relative frequency grid $v=f / f_{\mathrm{m}}$ when ionospheric parameters change [Kurkin et al., 1993; Ponomarchuk et al., 2016]. For predictive ionospheric parameters on the frequency grid, we compute DFC of BS signals along the leading edge. The predictive DFC of BS signal $P_{\mathrm{m}}(f)$ is recalculated into the relative frequency grid $v$. As the initial frequency $f_{\mathrm{m}}$ we take MUF for the maximum signal range. After the secondary processing of the experimental BS ionogram, there is a matrix of experimental points, which corresponds to a two-dimensional array of the group path $P_{i}$ on the frequency grid $f_{j}$ for points with significant amplitude. The experimental points are also transferred to the relative frequency grid $v$. The algorithm for automatically identifying propagation modes on BS ionograms involves determining the maximum of the histogram of experimental points falling into the model mask constructed from the long-term forecast, with $f_{\mathrm{m}}$ changing at the relative frequency grid $v=f / f_{\mathrm{m}}$. By multiplying $v$ by the computed value of the real $f_{\mathrm{m}}$, the predictive DFC values are transferred to the frequency scale. Thus we restore and identify DFC of BS signals for missing sounding frequencies.

Figure 1 shows a BS ionogram obtained on January 22, 2007 at 06:31 UT. The main trace of a BS signal reflected from the F2 layer is a continuation of the height-frequency characteristic of the second-order mode under quasi-vertical signal propagation from the transmitter to the receiver, beginning from the critical frequency of the F2 layer. On the ionogram, there are multiple signals of quasi-vertical sounding in the frequency range 4-7 MHz. Solid lines in the Figure indicate the results of calculation of DFC of BS signals along the leading edge $P_{\mathrm{m}}(f)$ for one-hop, two-hop, and three-hop propagation modes $1 \mathrm{~F} 2,2 \mathrm{~F} 2$ and $3 \mathrm{~F} 2$ respectively. $P_{\mathrm{m}}(f)$ has been simulated under the waveguide approach with the IRI model [Ponomarchuk et al., 2009; Ponomarchuk et al., 2014; Bilitza, Reinisch, 2008]. The $P_{\mathrm{m}}(f)$ simulation results for $1 \mathrm{~F} 2$ enables reliable identification of respective BS signals on the ionogram. There are BS signals corresponding to $2 \mathrm{~F} 2$ and $3 \mathrm{~F} 2$ on the ionogram, but they have low amplitude, therefore they are not extracted in the secondary data processing. Figure 2 presents the results of the secondary processing and interpretation of the 1F2 mode.

Then, we analyze the experimental data using the simulation results and automatic processing and interpretation of BS ionograms

\section{ANALYSIS OF EXPERIMENTAL DATA}

The analysis of the ionospheric sounding results by the BS method for a long time has revealed the most characteristic types of ionograms depending on the time of day, season, direction of sounding, and propagation medium conditions. BS ionograms can conventionally be divided into eight main types [Kabanov, Osetrov, 1965; Chernov, 1971; Altyntseva et al., 1990].

Type 1. Ionograms of the first type feature a small scattering of radio waves reflected by the $F$ layer along the entire path of their propagation, except for scattering by Earth's surface. Ionospheric conditions characterized by DFC of this type allow for selection and correction of operating frequencies. As a rule, we can obtain qualitative ionograms for distances up to $3000-4000 \mathrm{~km}$.

Figure 3 shows the BS ionogram obtained on November 12, 2009 at 01:40 UT. The central azimuth of sounding and receiving sector corresponds to $55^{\circ}$. Dots 


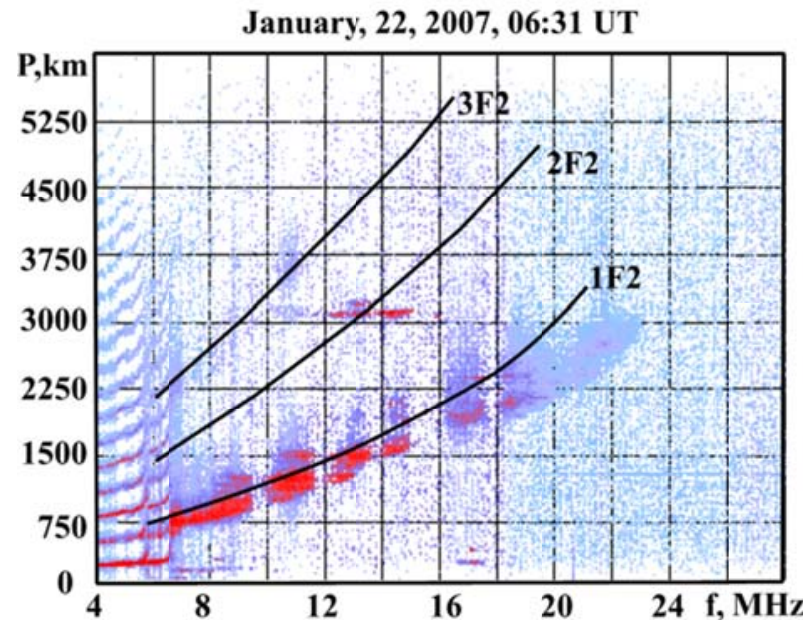

Figure 1. BS ionogram and simulation results $P_{\mathrm{m}}(f)$ : January $22,2007,06: 31 \mathrm{UT}$

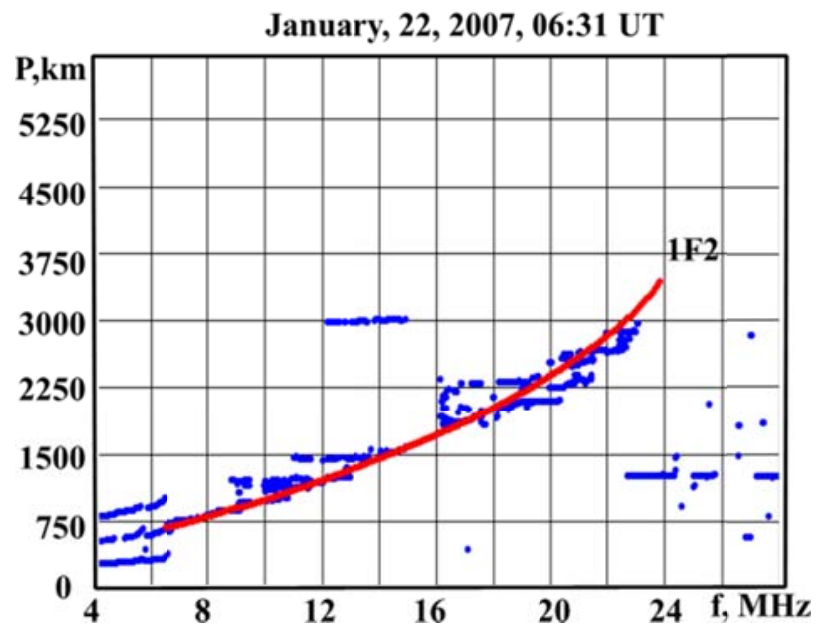

Figure 2. BS ionogram; processing and interpretation results: January 22, 2007, 06:31 UT

on the ionogram indicate BS signals with significant amplitude, which were found in the secondary processing of experimental data. The results of interpretation of the 1F2 mode (solid line in Figure 3) identify with confidence respective BS signals on the ionogram. Figure 4 presents the BS ionogram obtained on February 07, 2007 at 02:43 UT. It demonstrates that the processing and interpretation results also correctly identify the basic BS signal characteristics for $1 \mathrm{~F} 2$. Isolating BS signal tracks on the ionogram allows us to select and adjust operating radiocommunication frequencies [Ponomarchuk et al., 2016].

Type 2. Ionograms of this type occur when frequencies reflected by the $\mathrm{E}$ or $\mathrm{E}_{\mathrm{s}}$ layers are sufficiently high; in this case there may be no reflections from the $\mathrm{F}$ layer.

Ionograms of this type allow us to judge the sun lighting of Earth's surface at distances not exceeding $1500-2000 \mathrm{~km}$.

Figure 5 shows the BS ionogram obtained in the summer of 2005: June 15, 2005, 05:30 UT. The solid line in the Figure indicates the simulation results for DFC of BS signal along the leading edge $P_{\mathrm{m}}(f)$ of the $1 \mathrm{E}$ mode, acquired at an azimuth of $55^{\circ}$. There are no reflections from the $\mathrm{F}$ layer on the $\mathrm{BS}$ ionogram at the given
November, 12, 2009, 01:40 UT, azimuth 55

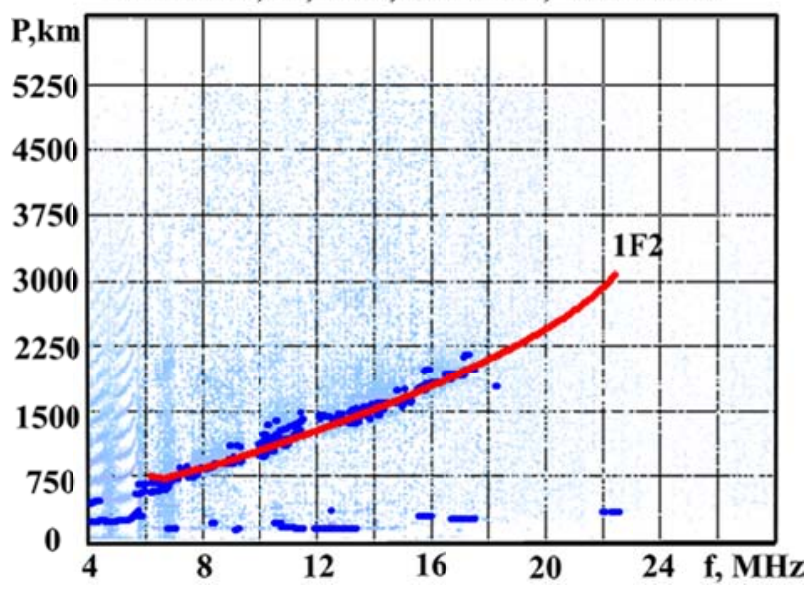

Figure 3. The same as in Figure 2 for November 12, 2009, 01:40 UT

February, 07, 2007, 02:43 UT, azimuth 55

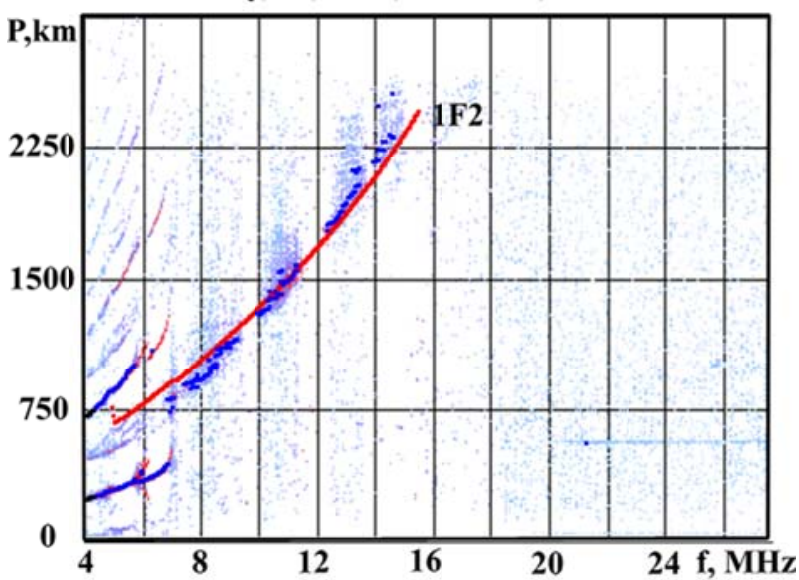

Figure 4. The same as in Figure 2 for February 07, 2007, 02:43 UT

time because of the strong absorption of the signal field in the distributed $\mathrm{E}$ and $\mathrm{E}_{\mathrm{s}}$ layers in the sounding sector.The presence of the sporadic $\mathrm{E}_{\mathrm{s}}$ layer in this period is confirmed by the experimental data of oblique sounding over the Magadan-Tory path, as well as by vertical sounding data. The results of BS signal DFC simulation along the leading edge of the propagation mode $1 \mathrm{E}$ differ significantly from the experimental data. This is associated with the error in predicting critical frequencies of the E layer in the IRI model. Figure 6 shows the results of the secondary processing and interpretation of signals corresponding to the propagation mode 1E. Figure 7 exemplifies an experimental BS ionogram for the case when signals scattered by Earth's surface and reflected from the $\mathrm{E}$ and $\mathrm{E}_{\mathrm{s}}$ layers arrive at the receiving point. The results of data processing and interpretation are presented in Figure 8. Before the operating sounding frequency of $20 \mathrm{MHz}$, scattered signals are reflected by the E layer; after $20 \mathrm{MHz}$, by the $\mathrm{E}_{\mathrm{s}}$ layer.

Type 3. A signal arrives at the receiving point along two-hop or even three-hop trajectories, being reflected by the F layer and Earth's surface. Combined modes can also be involved in BS signal formation. With a certain choice of transmitter parameters (rate of frequency 
June, 15, 2005, 05:30 UT, azimuth 55

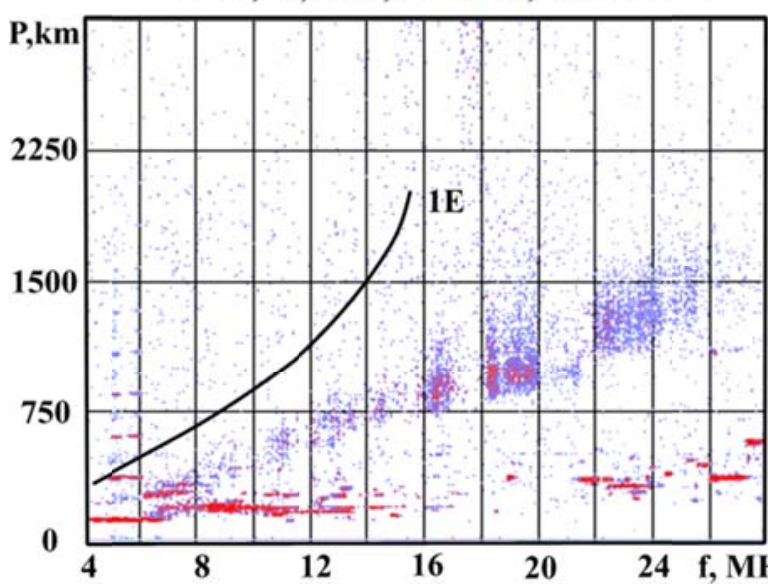

Figure 5. BS ionogram and simulation results $P_{\mathrm{m}}(f)$ : June 15, 2005, 05:30 UT

June, 15, 2005, 05:30 UT, azimuth 55

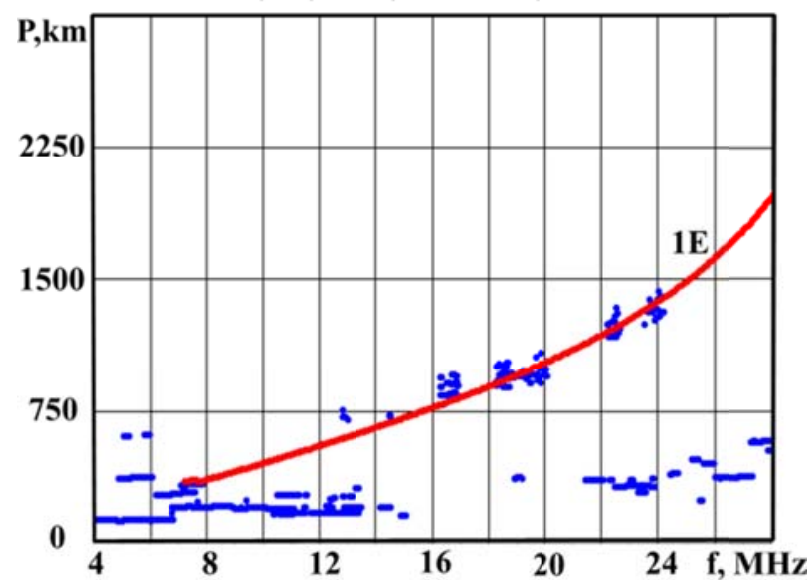

Figure 6. BS ionogram; processing and interpretation results: June 15, 2005, 05:30 UT

change, transmitter power), DFC of BS signals can be formed by scattering areas located at a distance up to 10 $000 \mathrm{~km}$ [Altyntseva et al., 1990].

Type 4. This type includes BS ionograms such that in addition to signals coming from the main radiation direction there are signals coming from other directions.

Type 5. Ionograms of this type exhibit atypical traces of reflections having a weak frequency dependence of the group path, which cannot be interpreted as typical BS signals. The appearance of such signals may be associated with the reflection from strong reflectors such as mountain ridges or land-sea boundary.

As an illustration of type 3, 4, and 5 BS ionograms, we present the experimental data acquired with the ISTP SB RAS chirp ionosonde on January 22, 2007. The purpose of the experiment was to study features of formation of scattered BS signals coming from different azimuth directions. The BS signals were received with the BS-2 antenna array, with sequential switching of azimuths of the main receiving direction every $15 \mathrm{~min}$ within an hour: $55^{\circ}, 121^{\circ}, 235^{\circ}$, and $301^{\circ}$. Figures $9-13$ show the experimental BS ionograms and the results of simulation of BS DFC along the leading edge $P_{\mathrm{m}}(f)$. The ionogram obtained for the 05:01 UT sounding session
June, 15, 2005, 03:45 UT, azimuth 55

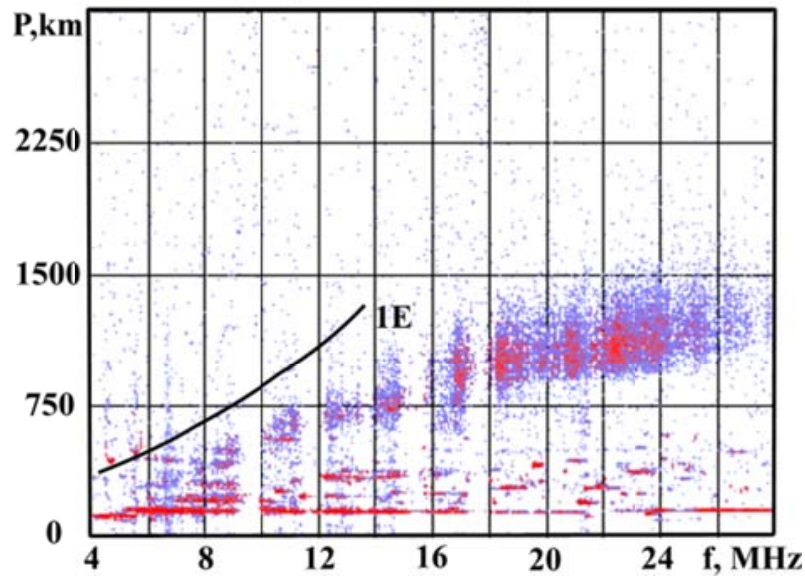

Figure 7. BS ionogram and simulation results $P_{\mathrm{m}}(f)$ : June 15, 2005, 03:45 UT

June, 15, 2005, 03:45 UT, azimuth 55

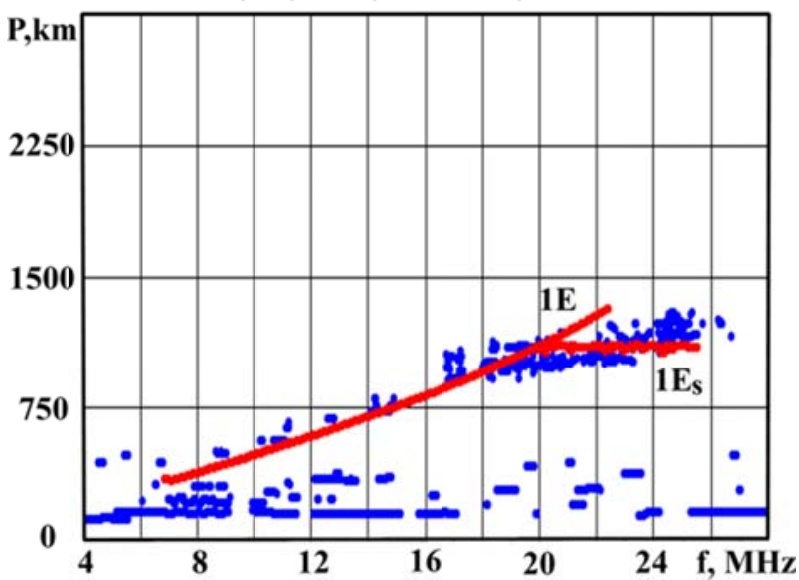

Figure 8. BS ionogram; processing and interpretation results: June 15, 2005, 03:45 UT

(Figure 9) is a type $4 \mathrm{BS}$ ionogram such that in addition to signals coming from the main radiation direction there are signals coming from other directions. The results of the BS DFC simulation for the propagation mode 1F2 with the IRI model, which were obtained for the $55^{\circ}$ azimuth, are presented in Figure 9 (solid line). This Figure also shows the results of the simulation of $P_{\mathrm{m}}(f)$ (dashed line) obtained for the $235^{\circ}$ azimuth opposite to the main receiving direction of the BS-2 antenna array. The comparison between the simulation results and the experimental data leads to the conclusion that the separation of BS signal DFC for this session is due to the detection of the scattered signals by the back directional lobe of the receiving antenna. The slope of DFC of BS signals coming from the north direction is greater than that of DFC of signals coming from the south direction.

The effect of separation of the BS signal DFC is more pronounced during the transient hours of day along the sounding direction and becomes stronger with increasing transmitter power [Altyntseva et al., 1990]. A similar, less pronounced, separation of BS DFC is observed for the 05:46 UT session in the sounding sector with an azimuth of $301^{\circ}$. Figure 12 shows the BS 


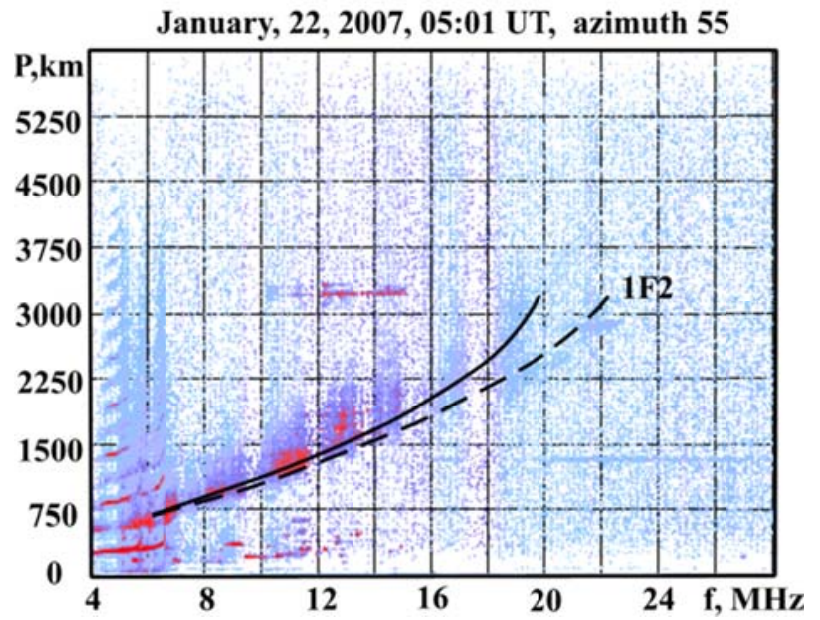

Figure 9. BS ionogram and simulation results $P_{m}(f)$ : January 22, 2007, 05:01 UT

January, 22, 2007, 05:16 UT, azimuth 121

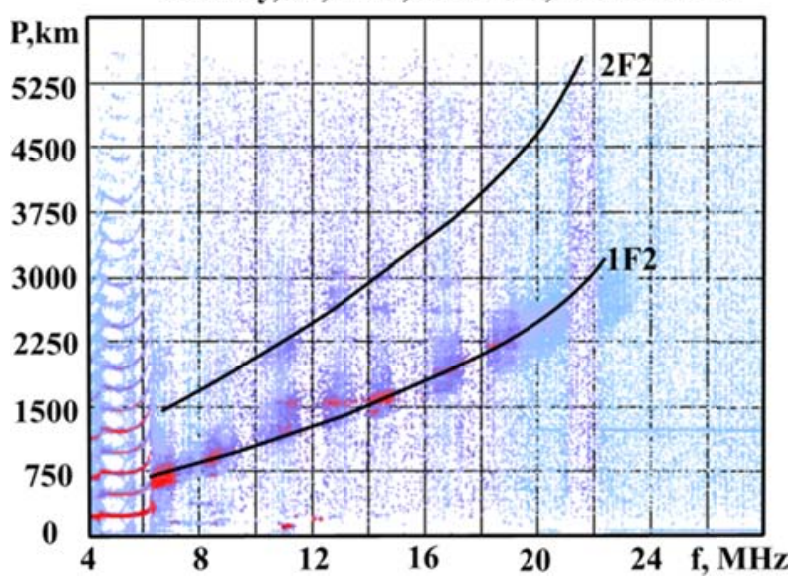

Figure 10. The same as in Figure 9 for 05:16 UT

January, 22, 2007, 05:31 UT, azimuth 235

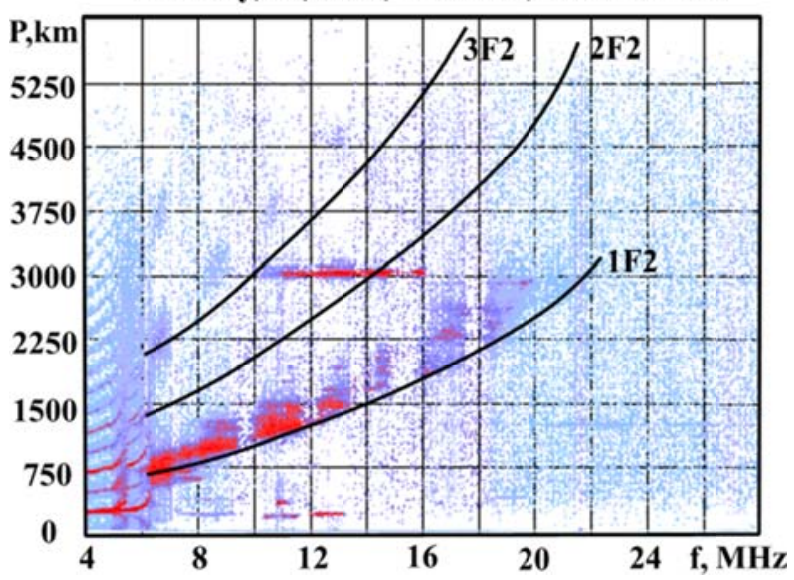

Figure 11. The same as in Figure 9 for 05:31 UT

ionogram obtained on January 22, 2007 at 05:46 UT, and the results of BS DFC simulation along the leading edge. Solid lines are the results of the $P_{\mathrm{m}}(f)$ simulation in the BS sector with an azimuth of $301^{\circ}$; dashed lines, with an azimuth of $121^{\circ}$.

In this case, the BS signal has higher amplitude and a wider frequency interval than the direct signal.

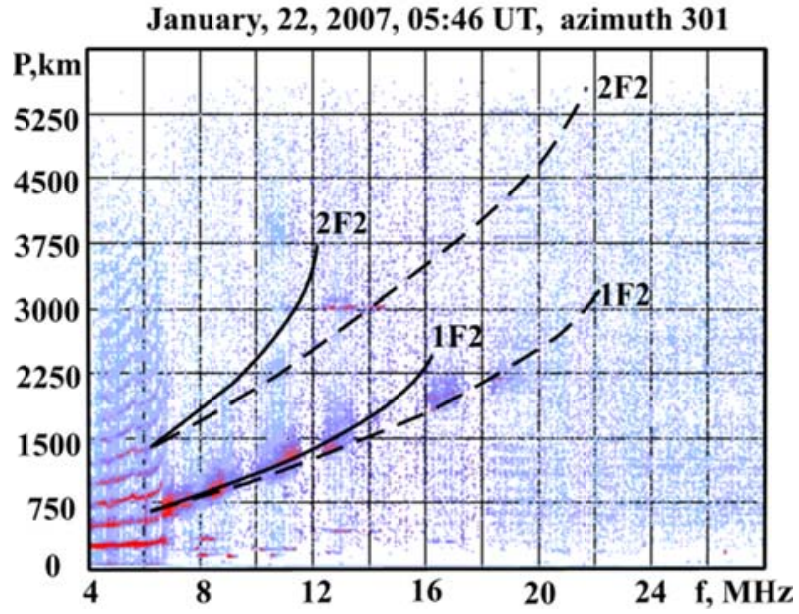

Figure 12. The same as in Figure 9 for 05:46 UT

In addition to the BS signals corresponding to the 1F2 mode, the ionograms shown in Figure 10-12 have signals arriving at the receiving point along two-hop and even three-hop trajectories through reflection from the F layer and Earth's surfacie. Therefore, these ionograms fall into type 3 too. The two-hop and three-hop BS signals are well identified from the results of BS DFC simulation based on the normal-wave method with the use of the IRI model, without adapting ionospheric parameters.

The BS ionogram shown in Figures 9 and 11 can also be assigned to the type of ionograms with atypical traces of reflections having a weak frequency dependence of the group path, which cannot be interpreted as typical BS signals. The appearance of such signals on the ionograms for sounding sessions at 05:01 and 05:31 UT may be related to the reflection from mountain ridges in the south of Central Asia - the Pamir mountain system located at a distance of $\sim 3000 \mathrm{~km}$ from the transmission point in the direction with an azimuth of $235^{\circ}$.

Type 6 . The radio wave propagation conditions vary considerably along the sounding direction, thus hindering selection of operating frequencies. Figure 13 shows the BS ionogram obtained in the summer of 2005: June 15, 2005, 13:15 UT. The solid line indicates the results of the BS DFC simulation in the leading edge $P_{\mathrm{m}}(f)$ of the $1 \mathrm{~F} 2$ mode for the sounding direction with an azimuth of $55^{\circ}$; the dashed line, for the reverse direction with an azimuth of $235^{\circ}$. Reflections from the $\mathrm{F}$ layer on the BS ionogram at the given time are present up to $14 \mathrm{MHz}$ for the direct signal and up to $18 \mathrm{MHz}$ for the reverse signal. For frequencies over $18 \mathrm{MHz}$ there are no signals corresponding to the $1 \mathrm{~F} 2$ mode on the ionogram, as evidenced by the results of the BS DFC simulation in the leading edge with the IRI model. The presence of the sporadic $E_{\mathrm{s}}$ layer in the sounding sector allows us to detect BS signals reflected from the $\mathrm{E}_{\mathrm{s}}$ layer. Figure 14 shows the results of the secondary processing and interpretation of BS signals corresponding to the propagation modes $1 \mathrm{~F} 2$ and $1 \mathrm{E}_{\mathrm{s}}$. The solid line indicates the results of interpretation of BS signals arriving at the receiving point from the $55^{\circ}$ azimuth; the dashed line, the results of interpretation of signals coming from the reverse direction with the $235^{\circ}$ azimuth. 
June, 15, 2005, 13:15 UT, azimuth 55

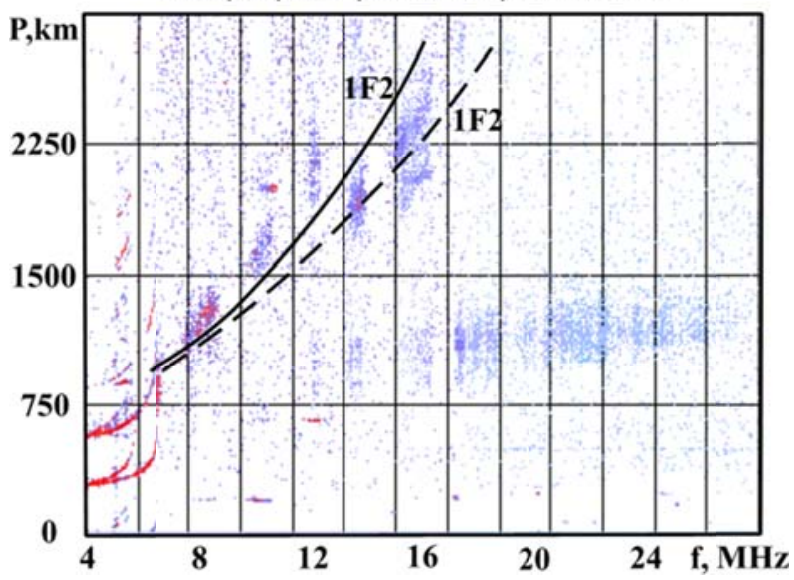

Figure 13. BS ionogram and simulation results $P_{m}(f)$ : June 15, 2005, 13:15 UT

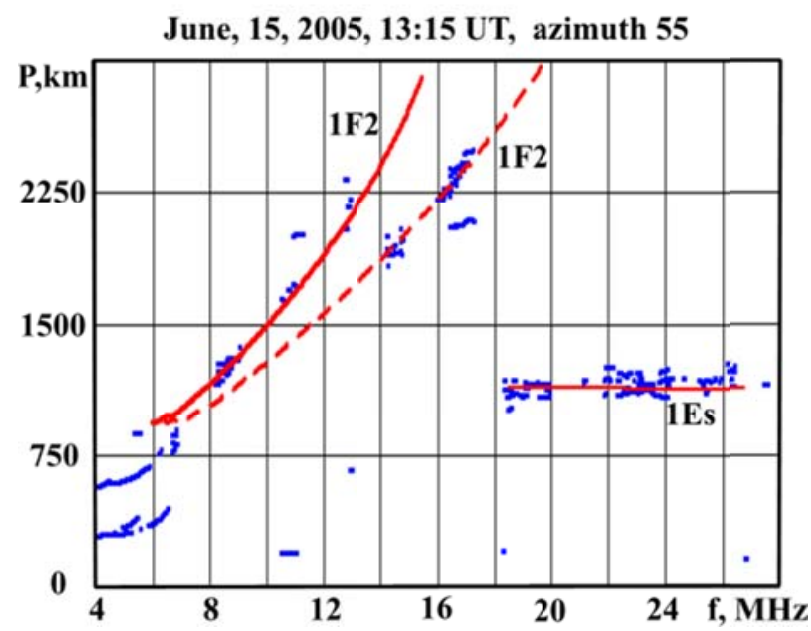

Figure 14. BS ionogram; processing and interpretation results: June 15, 2005, 13:15 UT

Type 7. In the ionosphere there are no conditions for regular signal reflection and there are considerable irregularities. On ionograms there are only backscattering signals from ionospheric irregularities. This generally occurs during strong ionospheric disturbances. Figure 15 presents the BS ionogram obtained on September 11, 2005 at 02:10 UT during the strong magnetic storm of September 11-16, 2005. Ionograms of this type feature disturbances of the radio wave propagation medium during geomagnetic storms. BS data cannot be used to forecast radiocommunication conditions.

Type 8 . Type 8 ionograms feature signals for which boundaries of reflections are independent of the sounding frequency and are approximately equal to 100 $\mathrm{km}$. Similar reflected signals are detected in the case of a complete screening of the upper ionospheric layers by the $E_{s}$ layer. A sign of ionograms of this type is the presence of signals coming from ranges of one hundred to several hundred kilometers. As an example, Figure 16 shows the BS ionogram received on November 11, 2009 at 13:30 UT. Ionograms of this type also preclude using sounding data to control radiocommunications.
September, 11, 2005, 02:10 UT, azimuth 55

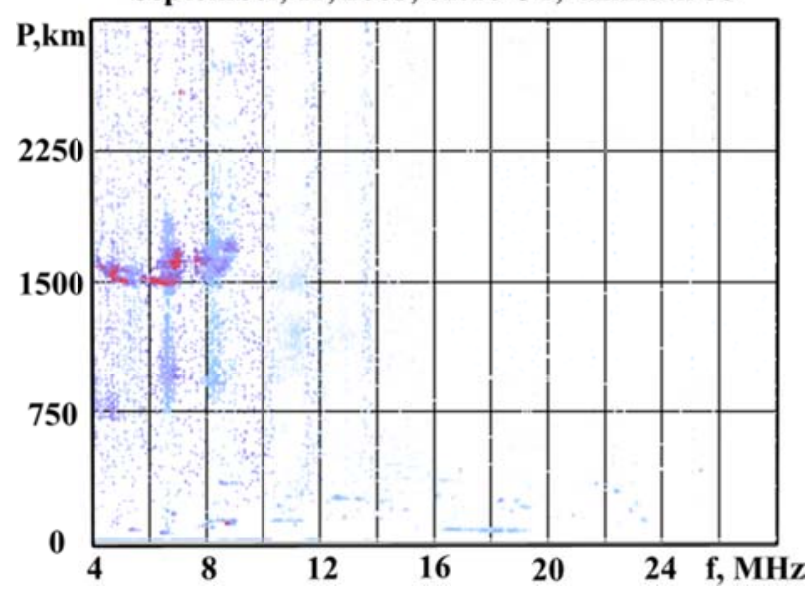

Figure 15. BS ionogram: September 11, 2005, 02:10 UT

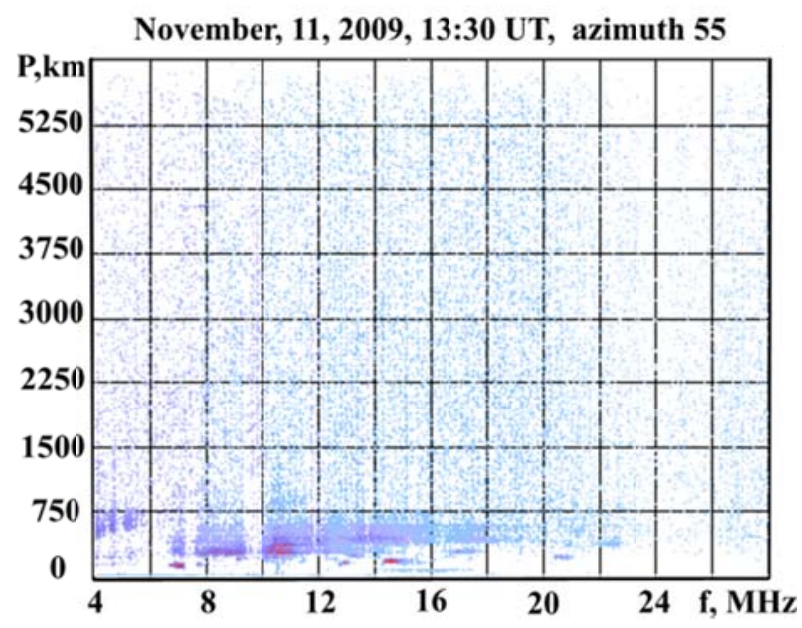

Figure 16. BS ionogram: November 11, 2009, 13:30 UT

\section{CONCLUSION}

The accumulated dataset enables us to examine features of BS signal propagation under various heliogeo- physical conditions. We have identified the most characteristic types of ionograms and have determined conditions for a particular type depending on the time ofday, season, direction of sounding, and propagation medium conditions. In winter, spring, and autumn, the prevailing types of ionograms are those with BS signals corresponding to the one-hop mode of propagation through reflection from the F layer. Ionospheric conditions characterized by ionograms of this type create the most favorable conditions for selecting and adjusting operating oblique sounding frequencies. It is generally possible to obtain qualitative ionograms for distances up to 3000 $4000 \mathrm{~km}$. During transient hours of day, along with the main signals there are signals arriving from directions opposite to the sounding azimuth. In summer, there are signals reflected by $\mathrm{E}$ or $\mathrm{E}_{\mathrm{s}}$ layers. At the same time, frequencies of received signals are high enough, and there may be cases in which there are no reflections from the $\mathrm{F}$ layer. Ionograms of this type allow us to judge the sun lighting of Earth"s surface at distances not exceeding $1500-2000 \mathrm{~km}$. We have also obtained iono- 
grams with atypical reflection tracks having a weak frequency dependence of the group path, which cannot be interpreted as typical BS signals.

The work was supported by Program for Fundamental Research of the Russian Academies of Sciences No. II.12.2.2 and by RFBR grant No. 16-32-00788. Experimental data have been acquired with the chirp ionosonde of ISTP SB RAS.

\section{REFERENCES}

Altyntseva V.I., Brynko I.G., Galkin I.A., et al. Oblique backscatter ionospheric sounding by a linear-frequency modulation signal. Issledovaniya po geomagnetizmu, aeronomii $i$ fizike Solntsa [Research on Geomagnetism, Aeronomy, and Solar Physics]. Moscow, Nauka Publ., 1990, iss. 92, pp. 106116. (In Russian).

Bilitza D., Reinisch B.W. International Reference Ionosphere 2007: Improvements and new parameters. $A d v$. Space Res. 2008, vol. 42, pp. 599-609.

Brynko I.G., Galkin I.A., Grosov V.P., et al. An automatically controlled data gathering and processing system using an FMCW ionosonde. Adv. Space Res. 1988, vol. 8, no. 4, pp. 121-124.

Chernov Yu.A. Vozvratno-naklonnoe zondirovanie ionosfery [Backscatter Ionospheric Sounding]. Moscow, Svyaz 'Publ., 1971, 204 p. (In Russian).

Kuk Ch., Bernfel'd M. Radiolokatsionnye signaly. Teoriya i primenenie [Radar Signals: Theory and Application]. Moscow, Sovetskoe radio Publ., 1971, 567 p. (In Russian). English edition: Cook C., Bernfeld M. Radar Signals: An Introduction to Theory and Application. Academic press, 1967, $567 \mathrm{p}$.

Dyson P.L. A simple method of backscatter ionogram analysis. J. Atmos. Terr. Phys. 1991, vol. 53, no. 1. pp. 75-88.

Earl G.F., Ward B.D. The frequency management system of the Jindalee over-the-horizon backscatter HF radar. Radio sci. 1987, vol. 22, no. 2, pp. 275-291.

Filipp N.D., Blaunstein N.Sh., Eruhimov L.M., Ivanov V.A., Uryadov V.P. Sovremennye metody issledovaniya dinami-cheskikh protsessov v ionosfere. [Modern Methods of Investigation of Dynamic Processes in the Ionosphere]. Kishinev, Shtiintsa Publ., 1991, 288 p. (In Russian).

Grozov V.P., Ilyin N.V., Kotovich G.V., Ponomarchuk S.N. Software system for automatic interpretation of ionosphere sounding data. Pattern Recognition and Image Analysis. 2012, vol. 22, no. 3, pp. 458-463.

Grozov V.P., Kiselev A.M., Kotovich G.V., Mikhailov S.Ya., Ponomarchuk S.N. Software for processing and interpretation of ionograms obtained by digital chirp ionosonde. Geliogeofizicheskie issledovaniya [Heliogeophysical Research]. 2013, vol 4, pp. 75-85. (In Russian).

Ilyin N.V., Khakhinov V.V., Kurkin V.I., Nosov V.E., Orlov I.I., Ponomarchuk S.N. The theory of chirp-signal ionospheric sounding. Proc. ISAP'96. Chiba, Japan, 1996, pp. 689-692.

Ivanov V. A., Ryabova N.V., Shumaev V.V., Uryadov V.P. Forecasting and updating HF channel parameters on the basis of oblique chirp sounding. Radio Sci. 1997, vol. 32, no. 3, pp. 983988.

Ivanov V.A., Kurkin V.I., Nosov V.E., Uryadov V.P., Shumaev V.V. FMCW-ionosounder and its application in ionosphere research. Izvestiya vuzov. Radiofizika. [Radiophysics and Quantum Electronics]. 2003, vol. 46, no. 11, pp. 919 952. (In Russian).

Kabanov N.I., Osetrov B.I. Vozvratno-naklonnoe zondirovanie ionosfery [Backscatter Ionospheric Sounding]. Moscow, Svyaz’ Publ., 1965, 112 p. (In Russian).

Kurkin V.I., Nosov V.E., Ponomarchuk S.N., Savkov S.S.,
Chistyakova L.V. Metod of real-time diagnostics of HF radio channel. Issledovaniya po geomagnetizmu, aeronomii i fizike Solntsa [Research on Geomagnetism, Aeronomy, and Solar Physics]. Novosibirsk, 1993, iss. 100, pp. 168-188. (In Russian).

Kurkin V.I., Nosov V.E., Matyushonok S.M. The features of round-the-world signal propagation over the paths of the Russian chirp-sounder network during low and mild solar activity. Izvestiya vuzov. Radiofizika. [Radiophysics and Quantum Electronics]. 2000, vol. 43, no.10, pp.755-765. (In Russian).

Podlesnyi A.V., Brynko I.G., Kurkin V.I., Berezovsky V.A., Kiselyov A.M., Petukhov E.V. Multifunctional chirp ionosonde for monitoring the ionosphere. Geliogeofizicheskie issledovaniya [Heliogeophysical Research]. 2013, no. 4, pp. 24-31. (In Russian).

Ponomarchuk S.N, Kurkin V.I., Oinats A.V. The diagnostics of ionosphere and earth ground surface by backscatter sounding data. PIERS 2009 Moscow Proc. 2009, pp. 1307 1310.

Ponomarchuk S.N., Ilyin N.V., Penzin M.S. The model of radio wave propagation in $1-10 \mathrm{MHz}$ frequency range on the base of normal wave technique. Solnechno-zemnaya fizika [Solar-Terrestrial Physics]. 2014, iss. 25, pp. 33-39. (In Russian).

Ponomarchuk S.N., Grozov V.P., Kotovich G.V., Kurkin V.I., Penzin M.S. Automatic processing and interpretation of backscatter ionosphere sounding ionograms. Proc. SPIE. 2016, vol. 10035, 100351E. DOI: 10.1117/12.2248765.

Sinnott D.H. The Development of Over-the-Horizon Radar in Australia. Defence Science \& Technology Organisation Publ., 1988, 39 p.

Uryadov V.P., Vertogradov G.G., Vertogradova E.G. Spread-F radar observations in the midlatitude ionosphere using an ionosonde-radiodirection finder. Izvestiya vuzov. Radiofizika [Radiophysics and Quantum Electronics]. 2013, vol. 56, no. 1, pp. 1-11. (In Russian).

Valov V.A., Vertogradov G.G., Vertogradov V.G., et al. LFM ionosonde-radiodirection finder and its application in the ionospheric researches. Fizicheskie osnovy priborostroeniya [Physical Bases of Instrumentation]. 2012, vol. 1, no. 4(5), pp. $24-43$. (In Russian).

Varakin L.E. Teoriya slozhnykh signalov [Theory of Complex Signals]. Moscow, Sovetskoe Radio Publ., 1970, 376 p. (In Russian).

Vertogradov G.G., Uryadov V.P., Vertogradov V.G., et al. Chirp ionosonde-radiodirection finder as a new tool for studying the ionosphere and radio-wave propagation. Izvestiya vuzov. Radiofizika. [Radiophysics and Quantum Electronics]. 2013, vol. 56, no. 5, pp. 259-275. (In Russian).

Wise J.C. Summary of recent Australian radar developments. IEEE Aerospace and Electronic Systems Magazine. December 2004, pp. 8-10.

How to cite this article

Ponomarchuk S.N., Kurkin V.I., Penzin M.S. Features of backscatter ionospheric sounding as studied with a chirp ionosonde. 2017. Vol. 3 , iss. 3. P. 54-60. DOI: $10.12737 /$ stp-33201706 